\title{
Involvement of the Anterior Cingulate Cortex in the Expression of Remote Spatial Memory
}

\author{
Cátia M. Teixeira, ${ }^{1,2}$ Stephen R. Pomedli, ${ }^{1}$ Hamid R. Maei, ${ }^{1,3}$ Nohjin Kee, ${ }^{1}$ and Paul W. Frankland $d^{1,3,4}$ \\ ${ }^{1}$ Program in Neuroscience and Mental Health, The Hospital for Sick Children, Toronto, Ontario, Canada M5G 1X8, ${ }^{2}$ Graduate Program in Areas of Basic and \\ Applied Biology, Universidade do Porto, 4050-345 Porto, Portugal, and ${ }^{3}$ Department of Physiology and 4 Institute of Medical Science, University of Toronto, \\ Toronto, Ontario, Canada M5S1A8
}

\begin{abstract}
Although the hippocampus plays a crucial role in the formation of spatial memories, as these memories mature they may become additionally (or even exclusively) dependent on extrahippocampal structures. However, the identity of these extrahippocampal structures that support remote spatial memory is currently not known. Using a Morris water-maze task, we show that the anterior cingulate cortex (ACC) plays a key role in the expression of remote spatial memories in mice. To first evaluate whether the ACC is activated after the recall of spatial memory, we examined the expression of the immediate early gene, $c$-fos, in the ACC. Fos expression was elevated after expression of a remote ( 1 month old), but not recent $(1 \mathrm{~d}$ old $)$, water-maze memory, suggesting that ACC plays an increasingly important role as a function of time. Consistent with the gene expression data, targeted pharmacological inactivation of the ACC with the sodium channel blocker lidocaine blocked expression of remote, but spared recent, spatial memory. In contrast, inactivation of the dorsal hippocampus disrupted expression of spatial memory, regardless of its age. We further showed that inactivation of the ACC blocked expression of remote spatial memory in two different mouse strains, after training with either a hidden or visible platform in a constant location, and using the AMPA receptor antagonist CNQX. Together, our data provide evidence that circuits supporting spatial memory are reorganized in a time-dependent manner, and establish that activity in neurons intrinsic to the ACC is critical for processing remote spatial memories.
\end{abstract}

Key words: hippocampus; spatial learning; Fos; lidocaine; CNQX; memory consolidation

\section{Introduction}

Experiments using lesion, electrophysiological, genetic, and neuroimaging approaches have helped to establish that the hippocampus plays an essential role in the formation of spatial memories (O'Keefe and Nadel, 1978; Burgess et al., 2002; Morris et al., 2003; Nakazawa et al., 2004; Leutgeb et al., 2005). However, as spatial memories mature they may become additionally (or even exclusively) dependent on extrahippocampal structures. Perhaps the best evidence that spatial memories eventually become dependent on extrahippocampal structures comes from neuropsychological studies of two patients: E.P. and K.C. (Teng and Squire, 1999; Rosenbaum et al., 2000). These patients both suffered substantial damage to the medial temporal lobe that resulted in extensive (or almost complete) lesions of the hippocampus. As in other patients with hippocampal lesions (Smith and Milner, 1981; Cave and Squire, 1991; Maguire et al., 1996), E.P. and K.C. both have difficulty acquiring new spatial information.

Received March 11, 2006; revised May 28, 2006; accepted May 31, 2006

This work was supported by grants from the Canadian Institutes of Health Research and the EJLB Foundation. C.M.T. received support from the Graduate Program in Areas of Basic and Applied Biology and the Portuguese Foundation for Science and Technology. We thank Sheena Josselyn for comments on previous drafts of this manuscript, and Christy Cole and Adrienne Yeung for technical assistance.

Correspondence should be addressed to Paul W. Frankland, Program in Neuroscience and Mental Health, The Hospital for Sick Children, Toronto, Ontario, Canada M5G 1X8. E-mail: paul.frankland@sickkids.ca.

D0I:10.1523/JNEUROSCI.1068-06.2006

Copyright $\odot 2006$ Society for Neuroscience $\quad$ 0270-6474/06/267555-10\$15.00/0
However, memory for similar spatial information that was acquired in the remote past is largely spared (Teng and Squire, 1999; Rosenbaum et al., 2000). Although it is a matter of debate whether these remote, hippocampal-independent spatial memories retain as much detail as those in control subjects (Rosenbaum et al., 2004; Clark et al., 2005a,b), these studies nonetheless indicate that spatial memories may become, at least partially, supported by extrahippocampal structures over the course of time. The identity of extrahippocampal regions that may support remote spatial memory is currently not known.

Recently, the medial prefrontal cortex (mPFC) has been shown to play a key role in the expression of remote memory (Frankland and Bontempi, 2005). The medial prefrontal cortex consists of several highly interconnected regions, including the anterior cingulate cortex (ACC), and prelimbic and infralimbic cortices. These regions are reciprocally connected to sensory, motor, and limbic cortices (Uylings et al., 2003) and they are therefore ideally situated to integrate and synthesize information from a large number of different sources. Lesions of the mPFC (including the ACC and prelimbic cortex) disrupted expression of remote (month old), but not recent (day old), trace eye-blink conditioning memories (Takehara et al., 2003). Similarly, pharmacological inactivation of the ACC specifically disrupted expression of remote, but not recent, contextual fear (Frankland et al., 2004) and five-arm discrimination (Maviel et al., 2004) memories. To evaluate whether the ACC plays a similar role in spatial 
memory, we trained mice in the Morris water-maze task (Morris et al., 1982). Using two complementary approaches, our data suggest that the ACC plays a specific role in processing remote spatial information. First, we found that the ACC is activated after remote, but not recent, probe tests. Second, we found that inactivation of the ACC blocks expression of remote, but not recent, spatial memory.

\section{Materials and Methods}

Mice. Male offspring from a cross between C57BL/6NTacfBr [C57B6] and 129Svev [129] mice (Taconic Farms, Germantown, NY) were used in the majority of these experiments. These mice were bred in our colony at The Hospital for Sick Children, and were maintained on a $12 \mathrm{~h}$ light/dark cycle with access to food and water ad libitum. In one experiment we used C57B6 mice, purchased from Taconic Farms. All mice were at least 8 weeks of age at the start of experiments, and behavioral procedures were conducted during the light phase of the cycle. Experiments were conducted blind to the treatment condition of the mouse, and according to local animal care protocols.

Water maze and general training procedures. Behavioral testing was conducted in a circular water-maze tank $(120 \mathrm{~cm}$ in diameter, $50 \mathrm{~cm}$ deep), located in a dimly lit room. The pool was filled to a depth of $40 \mathrm{~cm}$ with water made opaque by adding white nontoxic paint. Water temperature was maintained at $28 \pm 1^{\circ} \mathrm{C}$ by a heating pad located beneath the pool. A circular escape platform (10 cm diameter) was submerged $0.5 \mathrm{~cm}$ below the water surface and located in the one of the quadrants. For visible platform experiments, a cylindrical cue $(4 \mathrm{~cm}$ diameter $\times 4 \mathrm{~cm})$ was added to the platform. The pool was surrounded by curtains, at least $1 \mathrm{~m}$ from the perimeter of the pool. The curtains were white, and had distinct cues painted on them.

Before the commencement of training, mice were individually handled for 2 min each day for 1 week. On each training day, mice received six training trials (presented in two blocks of three trials; interblock interval was $\sim 1 \mathrm{~h}$; intertrial interval was $\sim 15 \mathrm{~s}$ ). On each trial they were placed into the pool, facing the wall, in one of four start locations. The order of these start locations was pseudorandomly varied throughout training. The trial was complete once the mouse found the platform or $60 \mathrm{~s}$ had elapsed. If the mouse failed to find the platform on a given trial, the experimenter guided the mouse onto the platform. In these experiments, we used three different water maze training protocols. In the hidden version, the platform was submerged in a fixed location for all trials. In the visible/fixed version, the platform was marked by a visible cue and in a fixed location for all trials. In the visible/variable version, the platform was marked by cue, and its location was pseudorandomly varied on each trial between 4 different positions in the pool. In this version only, nonpatterned white curtains replaced the curtains containing distal cues to minimize reliance on spatial strategies. After the completion of training, spatial memory was assessed in a probe test. In this test, the platform was removed from the pool, and the mouse was allowed $60 \mathrm{~s}$ to search for it.

Behavioral data from training and the probe tests were acquired and analyzed using an automated tracking system (Actimetrics, Wilmette, IL). Using this software, we were able to record a number of parameters during training, including escape latency and swim speed. In probe tests, we quantified performance by measuring the amount of time mice searched the target zone $(23 \mathrm{~cm}$ in radius, centered on the location of the platform during training) versus the average of three other equivalent zones in other areas of the pool (Moser et al., 1993; Moser and Moser, 1998; de Hoz et al., 2004). These zones each represent $\sim 15 \%$ of the total pool surface. Two additional analyses were performed. First, to evaluate the precision of search patterns (Moser et al., 1993), we quantified exploration in three concentric circles (radii: $16 \mathrm{~cm}, 23 \mathrm{~cm}$, and $28 \mathrm{~cm}$ ) centered on the platform location. The regions represented by the inner, middle, and outer circles are of equal area (with each representing $\sim 7 \%$ of the total pool surface). Second, to evaluate thigmotaxic behavior, we calculated the amount of time mice spent in the peripheral region of the pool (the area within $6 \mathrm{~cm}$ of the wall) (Martin et al., 2005). This peripheral region represents $\sim 19 \%$ of the total pool surface.
Fos immunocytochemistry. In experiments examining Fos expression in the ACC, mice were perfused transcardially with $0.1 \mathrm{M}$ PBS and then $4 \%$ paraformaldehyde (PFA) 90 min after the completion of behavioral testing. Brains were removed, fixed overnight in PFA, and then transferred to $30 \%$ sucrose solution and stored at $4^{\circ} \mathrm{C}$. Fifty-micrometer coronal sections were cut and free-floating sections were prepared for immunocytochemistry using anti-Fos primary rabbit polyclonal antibody (1: 20000, Rabbit anti-Fos polyclonal IgG; Oncogene Research Products, Darmstadt, Germany) and a biotinylated goat anti-rabbit antibody (1: 2000, Biotin-SP-conjugated affiniPure Goat anti-rabbit IgG; Jackson ImmunoResearch, West Grove, PA) was used as a secondary. Staining was revealed using the avidin-biotin peroxidase method (Vectastain $A B C$ kit; Vector Laboratories, Burlingame, CA) coupled to diaminobenzidine as chromogen. Quantitative analysis of Fos-positive nuclei was performed using the Image J processing system. Structures were anatomically defined according to a mouse brain atlas (Paxinos and Franklin, 2000). Because we found no evidence for lateralization of Fos expression in the ACC in our initial analyses (left, $7.72 \pm 0.94$; right, $7.14 \pm 1.11 ; F_{(1,5)}=$ $1.35 ; p>0.05$ ), immunoreactive neurons were counted unilaterally along the entire anterior-posterior extent of this region. In addition, Fos expression was quantified in subregions of the hippocampus (CA1, CA3, and dentate gyrus).

Surgery and pharmacological inactivation. Under chloral hydrate anesthesia and using standard stereotaxic procedures, stainless-steel guide cannulas (22 gauge) were implanted into the ACC $(+0.8 \mathrm{~mm}, 0 \mathrm{~mm}$, $-1.0 \mathrm{~mm}$ ) or the dorsal hippocampus (dHPC) $(-2.0 \mathrm{~mm}, \pm 2.0 \mathrm{~mm}$, $-2.0 \mathrm{~mm}$ ). Mice were allowed to recover for at least 1 week after surgery. Ten minutes before probe tests, mice received $0.5 \mu \mathrm{l}$ infusions of either drug or vehicle (PBS) at a rate of $0.2 \mu \mathrm{l} / \mathrm{min}$. In the majority of experiments, we used lidocaine ( $4 \%$; Sigma, St. Louis, MO). In one experiment, we used 6-cyano-7-nitroquinoxaline-2,3-dione (CNQX, $3 \mathrm{~mm}$; Tocris, Ellisville, MO). The injection cannula (28 gauge) was left in place for 2 min after the infusion and the mice were returned to their home cages before testing.

Anatomical specificity is a critical issue when using pharmacological techniques to inactivate brain regions, and we used two approaches to evaluate both location and extent of infusion. First, in all mice cannula placement was verified histologically. At the completion of the experiments, mice were overdosed with chloral hydrate and perfused transcardially with saline, followed by $4 \%$ PFA solution to fix the brain tissue. Brains were removed, postfixed in 4\% PFA solution, and cut into $50 \mu \mathrm{m}$ coronal sections on a cryostat. Sections were mounted on slides, stained with neutral red, and cannula placements were examined under a light microscope. Second, we examined Fos expression after water-maze testing in a subset of mice treated with CNQX. Using the same quantification methods described above, the extent of inactivation in CNQX-treated mice was estimated by defining a region where Fos expression was reduced by $50 \%$ or more relative to PBS-treated mice. Using this method, we found that Fos expression was reduced in a roughly circular region $1.0-1.5 \mathrm{~mm}$ in diameter, below the tip of the injector (see Fig. $6 e-f$ ). This indicates that infusions were largely restricted to the ACC, with limited diffusion to neighboring structures.

Experimental procedures. In the first experiment, we examined whether spatial memory testing induced Fos expression in the ACC. In this experiment, separate groups of mice were trained in either the visible/fixed or the visible/variable version of the water maze for $5 \mathrm{~d}$. Then either $1 \mathrm{~d}$ (recent test: visible/fixed, $n=8$; visible/variable, $n=8$ ) or $30 \mathrm{~d}$ (remote test: visible/fixed, $n=8$; visible/variable, $n=8$ ) later they were given a probe test. In the second experiment, examining whether inactivation of the ACC or dHPC disrupted spatial memory expression, mice were trained in the hidden version of the water maze for $3 \mathrm{~d}$. Spatial memory was then probed $1 \mathrm{~d}$ (PBS, $n=13$; ACC, $n=10$; dHPC, $n=9$ ) or $30 \mathrm{~d}$ (PBS, $n=12$; ACC, $n=9$; dHPC, $n=10$ ) later. Roughly half of the mice in the PBS group received infusions into the ACC, whereas the others received infusions into the dHPC. There were no differences between these two groups, and so they were combined. In the third experiment, C57B6 mice were trained for $7 \mathrm{~d}$ in the hidden version of the water maze. Spatial memory was then tested either $1 \mathrm{~d}$ (PBS, $n=19$; ACC, $n=9$; dHPC, $n=7$ ) or $30 \mathrm{~d}$ (PBS, $n=23$; ACC, $n=8$; dHPC, $n=9$ ) later. 
a.

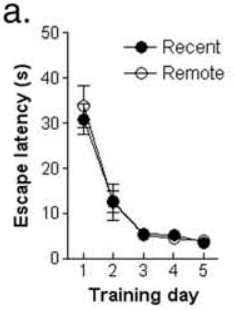

f.

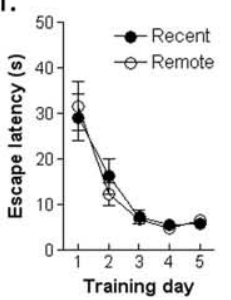

k.

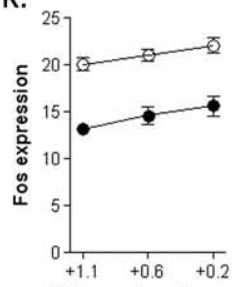

b.

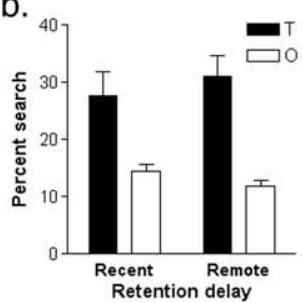

g.

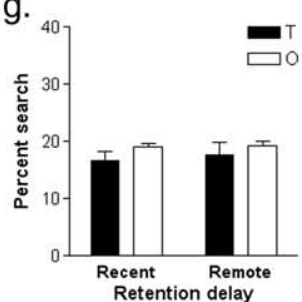

c.
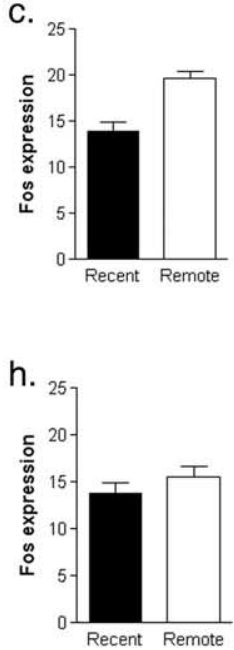

d.

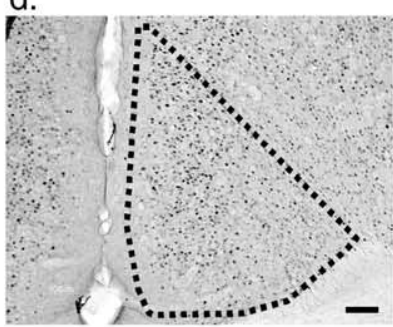

i.

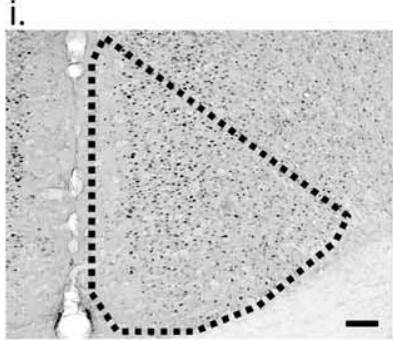

e.

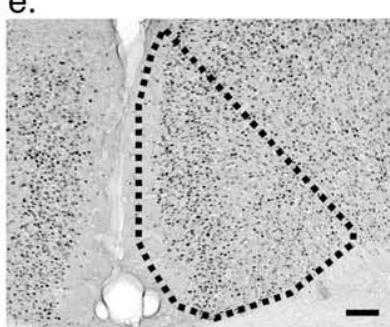

j.

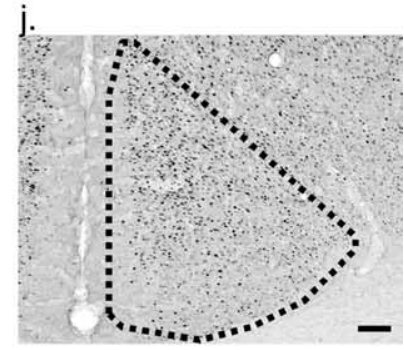

Distance from
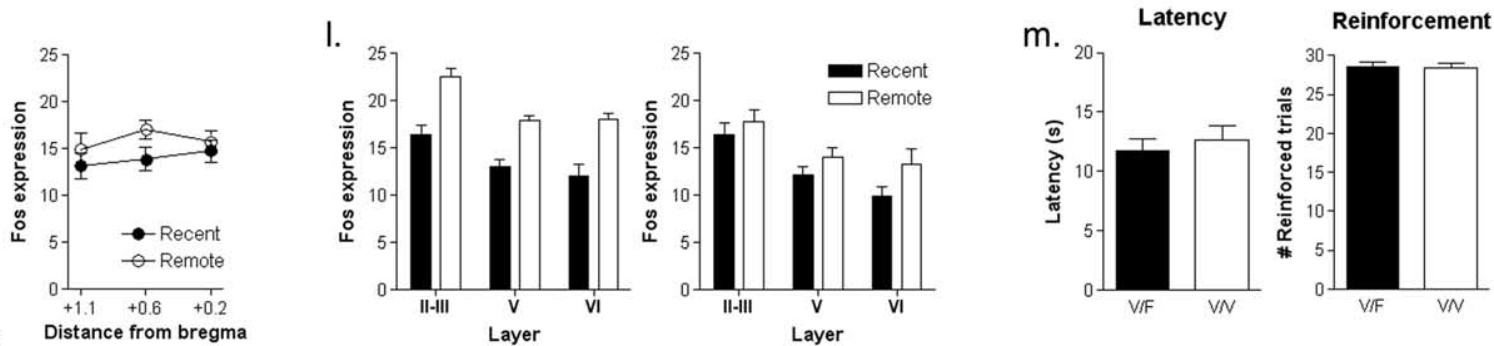

Figure 1. The ACC is activated after expression of remote spatial memory. $\boldsymbol{a}$, Mice were trained to locate a visible platform in a fixed location in the water maze. Over training, mice assigned to the recent or remote testing groups learned to find the escape platform with decreasing latencies. $\boldsymbol{b}$, Spatial memory was evaluated in a probe test either $1 \mathrm{~d}$ (recent group) or $30 \mathrm{~d}$ (remote group) after training. Both groups of mice spent more time searching the target zone $(T)$ compared with other $(0)$ zones in this test. $c$, Fos expression was elevated in the ACC after the remote compared with the recent probe test (number of Fos-positive nuclei per $10,000 \mu \mathrm{m}^{2}$ ). Representative sections show Fos expression in the ACC after a recent (d) and remote (e) probe test. Scale bars: $100 \mu \mathrm{m}$. $\boldsymbol{f}$, In the control condition, mice were trained to locate a visible platform, whose position varied from trial to trial. Over training, mice assigned to the recent or remote testing groups learned to find the visible platform with decreasing latencies. $\boldsymbol{g}$, Spatial memory was evaluated in a probe test either $1 \mathrm{~d}$ (recent group) or $30 \mathrm{~d}$ (remote group) after training. Neither group searched selectively in the target zone compared with other zones in these tests. $\boldsymbol{h}$, Levels of Fos expression in the ACC were equivalent after the recent and remote probe tests. Representative photomicrographs show Fos expression in the ACC after a recent (i) and remote (j) probe test. Scale bar, $100 \mu \mathrm{m}$. $\boldsymbol{k}$, Detailed analysis of Fos expression along rostral-caudal extent of the ACC in mice trained in the visible/fixed (left) and visible/variable (right) versions of the water maze. Distances relative to bregma are shown in millimeters. $I$, Detailed analysis of Fos expression in different layers in the ACC in mice trained in the visible/fixed (left) and visible/variable (right) versions of the water maze. $m$, Comparison of training performance in the visible/fixed (V/F) and visible/variable (V/V) versions of the water maze. During training there were no differences in the mean latency to find the escape platform (left) or the average number of reinforced trials (i.e., when mice successfully found the platform) (right). Error bars indicate SE.

Again, roughly half of the mice in the PBS group received infusions into the ACC, whereas the others received infusions into the dHPC. There were no differences between these two groups, and so they were combined. In the fourth experiment, mice were trained in the visible/fixed version of the water maze for $5 \mathrm{~d}$, and then their spatial memory was tested 30 d later after infusions of either PBS $(n=9)$ or lidocaine $(n=11)$ into the ACC. In the final experiment, mice were trained in the hidden version for $3 \mathrm{~d}$. Their spatial memory was assessed in a probe test $30 \mathrm{~d}$ later. Before this test, they received infusions of PBS $(n=7)$ or CNQX $(n=8)$ into the ACC. Four days later, they were given a second probe test, this time drug-free. After a further $20 \mathrm{~d}$, they received two more drug-free probe tests: one probe test was conducted with the visual distal cues present, another probe test with the visual distal cues obscured by adding a black curtain around the perimeter of the pool. The order of these tests counterbalanced.

\section{Results}

The ACC is preferentially activated after expression of remote spatial memory

The expression of the immediate early gene, $c$-fos, is correlated with levels of neuronal activity and can be used to track changes in the organization of memories at different times after learning (Kaczmarek and Robertson, 2002). Therefore, we first used Fos immunocytochemistry to evaluate whether the ACC is preferentially activated after expression of remote spatial memory. Mice were initially trained to find a visible platform in a fixed location in the pool, and then memory was assessed in separate groups of mice in probe tests either $1 \mathrm{~d}$ (recent test) or $30 \mathrm{~d}$ (remote test) later. Over the $5 \mathrm{~d}$ of training, mice required progressively less time to escape to the platform $\left(F_{(4,56)}=69.44 ; p<0.001\right)$ (Fig. $1 a)$, and there were no differences in escape latencies between mice assigned to the recent and remote groups [no significant effect of group $\left(F_{(1,14)}<1\right)$ or group by training day interaction $\left.\left(F_{(4,56)}<1\right)\right]$.

This training schedule produced a stable and long-lasting spatial memory; mice showed a strong spatial bias for the area of the pool where the platform was located during training in the recent and remote probe tests. We quantified this spatial bias by comparing how much time mice spent searching the target zone (a 23 $\mathrm{cm}$ radius circular zone centered on the former platform location) versus the average time they spent searching equivalent zones in the other three quadrants (Moser et al., 1993; Moser and Moser, 1998; de Hoz et al., 2004). ANOVA with group (recent vs remote) as a between-subjects variable and zone (target vs others) 
as a within-subjects variable revealed a significant main effect of zone $\left(F_{(1,14)}=\right.$ 21.96; $p<0.001)$, indicating that mice spent more time exploring the target zone compared with other zones. There was no significant effect of group $\left(F_{(1,14)}<1\right)$ or group by zone interaction $\left(F_{(1,14)}<1\right)$, indicating that mice performed similarly in the recent and remote tests (Fig. 1b). Although performance was similar in the recent and remote probe tests, we found that Fos expression in the ACC was significantly higher after the remote, compared with the recent, probe test $\left(F_{(1,13)}=27.39\right.$, $p<0.001$ ) (Fig. $1 c-e$ ). This increase was evident throughout the rostral-caudal extent of the ACC, and was not limited to a particular layer, or subset of layers, within the ACC (Fig. $1 k-l$ ).

These results suggest that the ACC is preferentially involved in the processing of remote (compared with recent) spatial information. However, mice in the remote test group also had considerably less recent experience of being handled, swimming in the water maze, and so on. Therefore, it is possible that increased Fos expression might be confounded by nonspecific increases in arousal associated with the remote probe test. To control for this, we trained a second group of mice in a visible/variable version on the water maze. As in the previous experiment, the platform was marked by a visible cue. However, because (1) the position of the platform was pseudorandomly varied between four possible locations on each trial, and (2) the curtains containing distal cues were replaced with featureless white curtains, this version of the water maze discourages the development of spatial strategies. When trained in this way, mice required progressively less time to escape to the platform $\left(F_{(4,56)}=34.62 ; p<0.001\right)$ (Fig. $1 f$ ), and there were no differences in escape latencies between mice assigned to the recent and remote groups [no significant group $\left(F_{(1,14)}<1\right)$ or group by training day interaction $\left.\left(F_{(4,56)}<1\right)\right]$. Consistent with the idea that training in the visible/variable version of the water maze discourages the development of a spatial bias, mice spent equivalent time searching in the target zone compared with other zones [no significant group $\left(F_{(1,14)}<1\right)$, zone $\left(F_{(1,14)}=1.34 ; p=0.27\right)$ or group by zone interaction $\left(F_{(1,14)}<\right.$ 1 )] in the probe tests either $1 \mathrm{~d}$ or $30 \mathrm{~d}$ after training (Fig. $1 g$ ). Most importantly, after this type of training, levels of Fos expression in the ACC were equivalent after the recent and remote probe tests $\left(F_{(1,13)}=1.11 ; p=0.31\right)$ (Fig. $\left.1 h-j\right)$. These results, therefore, indicate that the increased Fos expression after remote memory testing in the first experiment is most likely related to the remoteness of the spatial memory, and not to differences in arousal at the recent and remote time points.

It is worth noting that this visible/variable version of the water-maze controls for both swimming experience and reinforcement during the training period. Both the amount of time taken to swim to the platform (visible/fixed, $11.73 \pm 1.04$; visible/ variable, $\left.12.61 \pm 1.20 ; F_{(1,28)}<1\right)$, as well as the frequency of reinforcement (visible/fixed, $28.63 \pm 0.54$; visible/variable, $\left.28.38 \pm 0.63 ; F_{(1,28)}<1\right)$ were equivalent during training in the two versions (Fig. $1 \mathrm{~m}$ ). Other types of control conditions have been used, including swimming controls (Gusev et al., 2005). However, whereas swimming controls equate swimming experience, reinforcement differs between groups and this may lead to differences in motivation and/or stress levels. b.

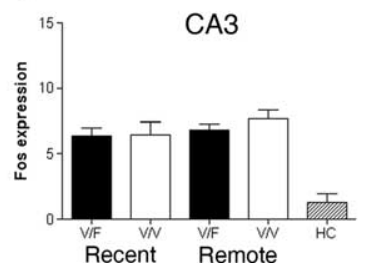

C.

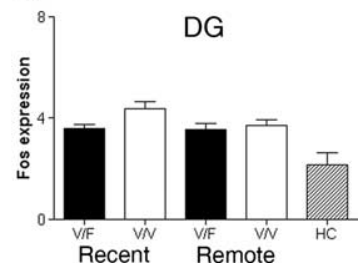

Figure 2. Fos expression in the hippocampus after recent and remote probe tests. Fos expression after recent and remote probe in CA1 (a), CA3 (b), and DG (c) after training in either the visible/fixed (V/F; black bars) or visible/variable (V/V; white bars) versions of the water maze (number of Fos-positive nuclei per 10,000 $\mu \mathrm{m}^{2}$ ). The hatched bars show Fos levels in the same regions ars indicate SE.

\section{Fos expression in the hippocampus after recent and remote probe tests}

We also examined Fos expression in the hippocampus after the recent and remote probe tests for mice trained in the visible/fixed and visible/variable versions of the water maze (Fig. 2). In each of the hippocampal subregions, Fos expression was similar in the recent and remote probe tests, regardless of the type of training: CA1 [no main effects of training $\left(F_{(1,27)}=2.26 ; p>0.05\right)$, retention $\left(F_{(1,27)}=2.10 ; p>0.05\right)$ or training by retention interaction $\left(F_{(1,27)}<1\right)$ ], CA3 [no main effects of training $\left(F_{(1,27)}<1\right)$, retention $\left(F_{(1,27)}=1.29 ; p>0.05\right)$ or training by retention interaction $\left(F_{(1,27)}<1\right)$ ], and dentate gyrus (DG) [no main effects of training $\left(F_{(1,27)}=3.80 ; p>0.05\right)$, retention $\left(F_{(1,27)}=2.17 ; p>\right.$ $0.05)$ or training by retention interaction $\left(F_{(1,27)}=1.56 ; p>\right.$ $0.05)]$. In every case, levels of Fos expression in these tested mice were significantly greater than those in home-cage controls $(n=$ 5 ; $t$ tests, all $p$ values $<0.05$ ), suggesting that performance (e.g., navigation), as well as mnemonic, aspects of water-maze testing contribute to activation of the hippocampus.

\section{Inactivating the ACC disrupts expression of remote spatial memory}

We next tested whether pharmacological inactivation of the ACC or dHPC disrupts expression of spatial water-maze memories. In this experiment, separate groups of mice were trained over $3 \mathrm{~d}$ to locate a hidden platform. During training, escape latencies declined over days (data not shown; $F_{(2,114)}=166.31 ; p<0.001$ ). After the completion of training, spatial memory was assessed in a probe test either $1 \mathrm{~d}$ (recent test) or $30 \mathrm{~d}$ (remote test) later. Mice treated with PBS before the probe test exhibited strong memory at both retention delays (Fig. $3 a$ ). ANOVA with group (recent vs remote) as a between-subjects variable and zone (target vs others) as a within-subjects variable was performed on the probe test data for the PBS-treated mice. This analysis revealed a significant main effect of zone $\left(F_{(1,23)}=40.62\right.$; $\left.p<0.001\right)$, indicating that mice searched selectively in the target zone that previously contained the escape platform. There was no significant group effect $\left(F_{(1,23)}=1.36 ; p=0.26\right)$ or group by zone $\left(F_{(1,23)}<\right.$ $1)$ interaction, indicating that PBS-treated mice performed similarly in the recent and remote tests. In contrast, lidocaine infusions into the ACC disrupted performance in the remote, but not recent, probe test (Fig. 3b). ANOVA revealed significant main effects of both group $\left(F_{(1,17)}=8.69 ; p<0.01\right)$ and zone $\left(F_{(1,17)}=\right.$ 15.65; $p<0.01)$ and, most importantly, a significant group by zone interaction $\left(F_{(1,17)}=9.04 ; p<0.01\right)$, indicating that mice spent more time searching the target zone (vs the other zones) in the recent, but not remote, probe test. Post hoc analyses (Neuman-Keuls; $p$ values $<0.05$ ) confirmed that lidocaine infusions into the ACC only disrupted performance in the $30 \mathrm{~d}$, and not the 
a.

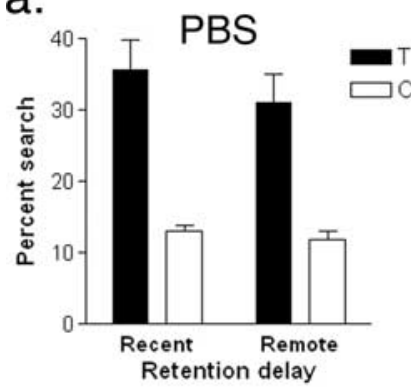

b.

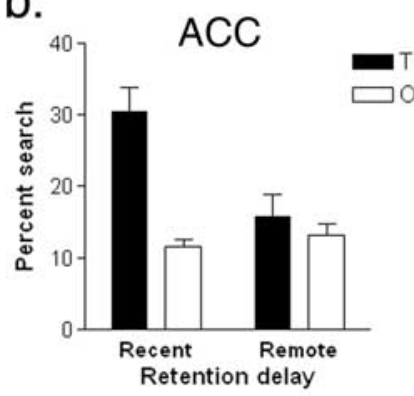

C.

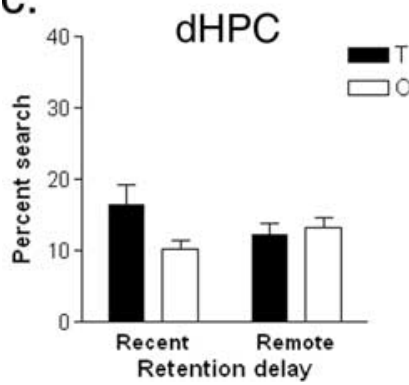

Figure 3. Effects of lidocaine inactivation of ACC and $\mathrm{dHPC}$ on recent and remote spatial memory. Spatial memory was evaluated in a probe test either $1 \mathrm{~d}$ (recent group) or $30 \mathrm{~d}$ (remote group) after training in the hidden version of the water maze. Ten minutes before testing, mice received infusions of either PBS $(\boldsymbol{a})$ or lidocaine into the $\mathrm{ACC}(\boldsymbol{b})$ or $\mathrm{dHPC}(\boldsymbol{c})$. Left, Percentage of time spent searching the target zone (T) compared with other $(0)$ zones in probe tests. Right, Density plot for grouped data showing where mice concentrated their searches in the recent and remote probe tests, respectively. The color scale represents the number of visits per animal per $5 \times 5 \mathrm{~cm}$ area. Error bars indicate $S E$.

$1 \mathrm{~d}$, probe test. Inactivation of the $\mathrm{dHPC}$ disrupted performance in both the recent and remote memory tests (Fig. $3 c$ ). ANOVA revealed no main effects of either group $\left(F_{(1,17)}<1\right)$ or zone $\left(F_{(1,17)}=2.77 ; p=0.11\right)$. Although there was a significant group by zone interaction $\left(F_{(1,17)}=5.41 ; p<0.05\right)$, post hoc analyses indicated that mice did not search significantly more in the target versus other zones in either the recent or remote probe tests (Neuman-Keuls; $p$ values $<0.05$ ). Finally, to examine the relative effects of inactivation of the ACC and dHPC on the expression of recent and remote spatial memory, we conducted ANOVA with infusion (PBS, ACC, and dHPC) as a betweensubjects variable and zone (target vs others) as a within-subjects variable. For the recent probe tests, these analyses revealed significant main effects of infusion $\left(F_{(2,29)}=11.66 ; p<0.001\right)$ and zone $\left(F_{(1,29)}=40.02 ; p<0.001\right)$, as well as a significant infusion by zone interaction $\left(F_{(2,29)}=3.92 ; p<0.05\right)$. Post hoc analyses revealed mice in the PBS and ACC groups spent significantly more time in the target zone than those in the dHPC group. For the remote probe tests, these analyses revealed significant main effects of infusion $\left(F_{(2,28)}=8.20 ; p<0.01\right)$ and zone $\left(F_{(1,28)}=\right.$ $11.38 ; p<0.01)$, as well as a significant infusion by zone interaction $\left(F_{(2,28)}=10.01 ; p<0.001\right)$. In this case, post hoc analyses revealed mice in the PBS groups spent significantly more time in the target zone than those in either the ACC or dHPC groups.

We next explored the nature of the deficits produced by these targeted inactivations in more detail. In initial training trials, naive mice tend to spend the majority of their time searching the periphery of the pool (Wolfer et al., 1998). This thigmotaxic behavior tends to decline rapidly over training (Fig. 4), suggesting that over time mice learn more effective search strategies (Martin et al., 2005). In the current study, we observed a similar decline in thigmotaxic behavior in mice in the PBS-treated group (Fig. 4a): the amount of time spent searching in the peripheral region of the pool (within $6 \mathrm{~cm}$ of the wall) declined dramatically from the first training trial (recent group, $57.27 \pm 2.63 \%$; remote group, $62.27 \pm 2.74 \%$ ) to the probe test (recent group, $4.11 \pm 3.11 \%$; remote group, $9.70 \pm 3.23 \%$ ) (main effect of training: $\left.F_{(1,23)}=264.34 ; p<0.001\right)$.

A similar decline occurred in mice in the ACC/lidocaine group (main effect of training: $F_{(1,17)}=114.33 ; p<0.001$ ) (Fig. $4 b$ ), suggesting that inactivation of the ACC does not interfere with memory for procedural aspects of the task. The decline was less pronounced in the remote memory group (training, $53.81 \pm 5.25 \%$; probe test, $18.79 \pm 3.82 \%)$. However, the time spent exploring this outer region in the probe is what would be predicted by a random search pattern because this outer region represents $\sim 19 \%$ of the total surface of the pool $\left(t_{(8)}=-0.04 ; p=0.97\right)$.

In the dHPC/lidocaine group, thigmotaxic behavior also declined from the first training trial to the probe test $\left(F_{(1,17)}=51.70 ; p<0.001\right)$ (Fig. $4 c)$. Again, these mice tended to spend more time exploring the periphery of the pool in the probe tests compared with PBStreated mice (recent, $32.26 \pm 6.56 \%$; remote, $24.85 \pm 6.22 \%$ ). Nonetheless, these values do not differ significantly from what would be expected for a random search pattern in either case (recent: $t_{(8)}=2.12, p=0.07$; remote: $t_{(9)}=0.90, p=0.39$ ). Together, these analyses suggest that these targeted inactivations have selective effects on spatial memory and spare memory for procedural aspects of the task (i.e., mice remember to avoid the walls).

In some tasks, inactivating or lesioning the dHPC produces temporally graded effects on memory, with recent memories affected more profoundly than remote memories (Squire and Alvarez, 1995; Squire et al., 2001; Frankland and Bontempi, 2005). Our analysis suggested inactivation of the dHPC has equivalent effects on performance in both the recent and remote probe tests. However, it is possible that inactivation of the dHPC results in less precise, but nonetheless partially spared, spatial preference in the remote test (Winocur et al., 2005). To evaluate whether there is any evidence of a graded effect, we examined the precision of search patterns in the recent and remote probe tests (Moser et al., 

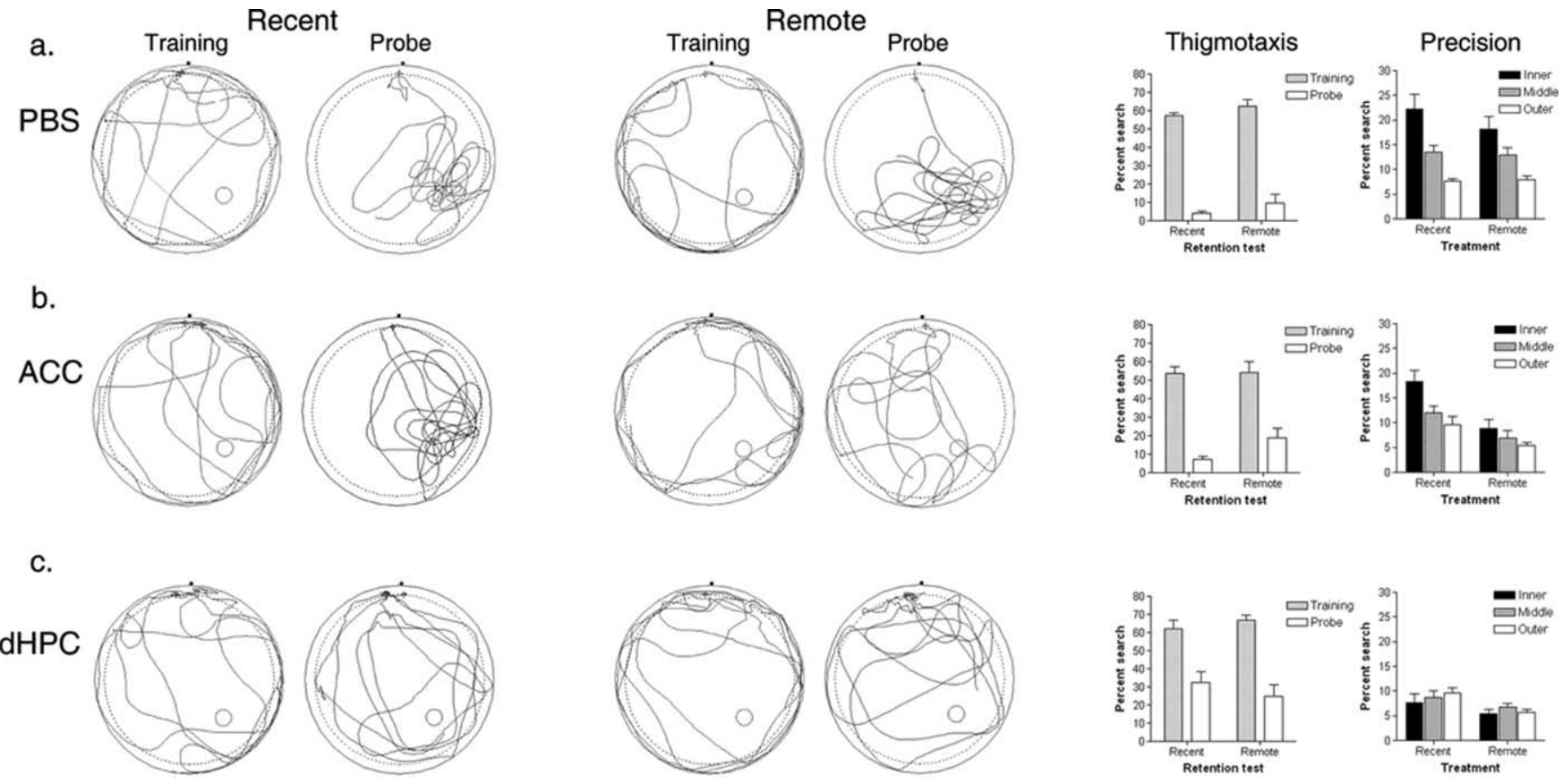

Figure 4. Detailed analysis of performance in recent and remote probe tests. Detailed analysis is shown for mice receiving infusions of PBS ( $(\boldsymbol{a})$ or lidocaine into the $A C C(\boldsymbol{b})$ or $\mathrm{dHPC}(\boldsymbol{c})$, respectively. Swim paths from the first training trial and probe tests are shown for representative mice in the recent and remote test groups, respectively. The position of the platform during training is marked in the lower right quadrant. In addition, a concentric circle indicates the peripheral region of the pool used to quantify thigmotaxic behavior. The graph on the left shows the amount of time spent in the periphery of the pool during the first training trial versus probe test for mice in the recent and remote test groups. The graph on the right shows the precision of search pattern indicated by time spent searching equal area regions marked by inner, middle, and outer circles centered on the position of the platform during training (for details, see Results). Error bars indicate SE.

1993). To do this we quantified exploration in three concentric circles (radii: $16 \mathrm{~cm}, 23 \mathrm{~cm}$ and $28 \mathrm{~cm}$ ) centered on the platform location. The areas represented by the inner, middle, and outer zones are of equal area, and more precise searches should produce more pronounced searching in the inner versus middle versus outer zones, respectively. PBS-treated mice had precise search patterns, spending more time exploring the inner versus middle versus outer zones, respectively, in the probe tests (significant main effect of zone: $F_{(2,46)}=42.73 ; p<0.001$ ) (Fig. $4 a$ ). There was no significant main effect of group $\left(F_{(1,23)}<1\right)$ nor group by zone interaction $\left(F_{(2,46)}=1.45 ; p=0.25\right)$, suggesting that searches were equivalently precise at both time points. Mice receiving lidocaine infusions into the ACC only showed this precise searching pattern in the recent probe test (Fig. $4 b)$. ANOVA revealed significant main effects of both group $\left(F_{(1,17)}=9.81 ; p<\right.$ $0.01)$ and zone $\left(F_{(2,34)}=20.76 ; p<0.001\right)$ and, most importantly, a significant group by zone interaction $\left(F_{(2,34)}=4.16 ; p<\right.$ $0.05)$, confirming that lidocaine infusions into the ACC only disrupted performance in the remote, and not the recent, probe test.

Analysis of probe test data in mice receiving lidocaine infusions into the dHPC provided no evidence for a graded effect on recent versus remote memory. Rather, mice spent equivalent amounts of time in each of the three zones in the probe tests (zone: $F_{(2,34)}=1.16 ; p=0.32$ ) (Fig. $4 c$ ). This was the case in both the recent and the remote probe test [no significant group by zone interaction $\left.\left(F_{(2,46)}<1\right)\right]$, suggesting that lidocaine inactivation of dHPC did not differentially affect the precision of recent versus remote spatial memory.

\section{Inactivation of the ACC disrupts expression of remote spatial memory in C57B6 mice}

Performance in learning tasks such as the Morris water maze is influenced by genetic background (Crawley et al., 1997). There- fore, to explore the generality of our findings, we also examined the effects of these same manipulations in a different mouse strain (inbred C57B6 mice). In this independent series of studies we found that lidocaine-induced inactivation of the ACC and dHPC produced exactly the same pattern of results (supplemental Fig. 1, available at www.jneurosci.org as supplemental material), suggesting that the roles of these regions in the expression of spatial memory are conserved across different genetic backgrounds.

\section{Inactivation of the ACC disrupts expression of remote spatial} memory after training with fixed, visible platform

In our initial immunocytochemical studies, mice were trained to find a fixed platform that was marked by a visible cue. So far, we only examined the effects of ACC inactivation on spatial memory expression after training in a hidden version of the water maze. Therefore, we next tested whether ACC inactivation impairs remote memory expression after training with a fixed, visible platform. During training, mice required progressively less time to escape to the platform $\left(F_{(4,72)}=74.69 ; p<0.001\right)$, and there were no differences in escape latencies between groups [no significant main effect of group $\left(F_{(1,18)}<1\right)$ or group by drug interaction $\left.\left(F_{(4,72)}<1\right)\right]$. Inactivation of the ACC with lidocaine disrupted performance in the remote memory probe test $30 \mathrm{~d}$ after training (Fig. 5). Whereas PBS-treated mice spent significantly more time exploring the target compared with other zones, this spatial bias was less pronounced in the lidocaine treated mice (significant group by zone interaction: $\left.F_{(1,18)}=5.64 ; p<0.05\right)$. Post hoc analyses showed that only the PBS-treated mice spent more time exploring the target zone compared with other zones $(p<0.05)$. These data indicate that inactivation of the ACC with lidocaine disrupts expression of remote spatial memory, regardless of whether the platform was visible or hidden during training. Im- 


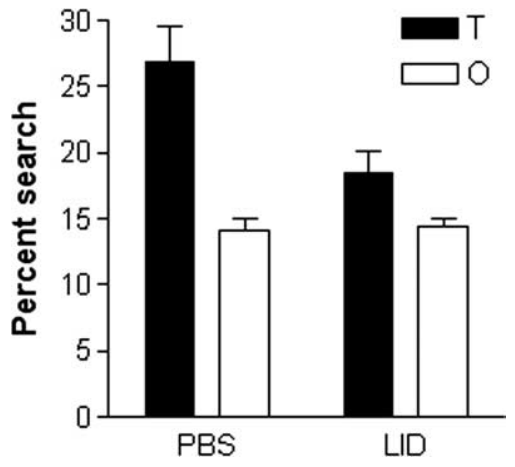

Figure 5. Effects of inactivating the ACC on remote spatial memory after training with fixed, visible platform. Spatial memory was evaluated in a probe test $30 \mathrm{~d}$ after training. The percentage of time spent searching the target zone $(\mathrm{T})$ compared with other $(0)$ zones in the probe test is shown for mice that received infusions of either PBS or lidocaine into the ACC. Error bars indicate SE.

mediately after the probe test, PBS- and lidocaine-treated mice were given a second test, where the escape platform was placed back into the pool and marked by a visible cue. In this test, both PBS- and lidocaine-treated mice found the platform quickly, and there was no difference in their escape latencies $\left(t_{(17)}=1.15 ; p=\right.$ 0.27; data not shown), indicating that lidocaine infusions did not interfere with sensory and motivational requirements of this task.

\section{Inactivation of the ACC with $\mathrm{CNQX}$ reversibly disrupts expression of remote spatial memory}

Lidocaine is a sodium channel blocker and therefore suppresses neuronal activity of both excitatory and inhibitory neurons in the ACC, as well as fibers of passage (Sandkuhler et al., 1987). To evaluate whether excitatory transmission is necessary for expression of remote spatial memories, we used the AMPA receptor antagonist, CNQX (Day et al., 2003). Mice were trained for $3 \mathrm{~d}$ in the hidden version of the water maze, and then tested $30 \mathrm{~d}$ later.
During training, mice learned to find the platform $\left(F_{(2,26)}=\right.$ 64.03; $p<0.001$ ), and there were no differences in escape latencies between mice assigned to the PBS and CNQX drug groups [no significant group $\left(F_{(1,13)}=2.08 ; p=0.17\right)$ or group by training day interaction $\left.\left(F_{(2,26)}=1.66 ; p=0.21\right)\right]$. CNQX infusion before the probe test blocked expression of remote spatial memory (significant group by zone interaction: $F_{(1,13)}=5.00 ; p<$ 0.05 ) (Fig. 6a), indicating that CNQX produces similar effects to lidocaine when infused into the ACC. Post hoc analyses confirmed that whereas PBS-treated mice searched selectively, CNQX-treated mice spent equivalent time searching the target versus other zones $(p<0.05)$. These results suggest that activity of intrinsic excitatory neurons in the ACC is critical for the expression of remote spatial memory.

CNQX infusion suppresses neural activity for $\sim 60 \mathrm{~min}$ (Day et al., 2003) and, therefore, these infusions should only transiently disrupt expression of spatial memory. To test whether the effects of the CNQX infusions were reversible, mice were tested drug-free $4 \mathrm{~d}$ later. In this test, both groups of mice (those previously treated with CNQX and those previously treated with PBS) searched selectively in the target area of the pool for the platform [significant main effect of zone: $F_{(1,13)}=26.31, p<0.001$; no significant group $\left(F_{(1,13)}=2.55 ; p=0.13\right)$, or group by zone interaction $\left(F_{(1,13)}=1.21 ; p=0.29\right)$ ] (Fig. 6b), indicating that the effects of CNQX were reversible, and likely did not produce any permanent damage in the ACC.

\section{Performance in remote probe tests depends on distal visual cues}

Targeted search strategies in the water maze depend on the formation of an accurate, allocentric representation of the distal cues surrounding the maze (O'Keefe and Nadel, 1978; Eichenbaum et al., 1990). To test whether remote spatial memory depended on these distal cues in our water maze, we performed two additional probe tests on the mice above with these distal cues either (1) present or (2) obscured by a black curtain. All mice a.

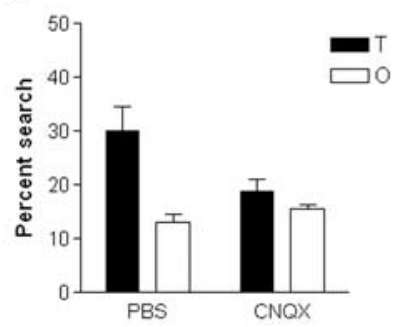

b.

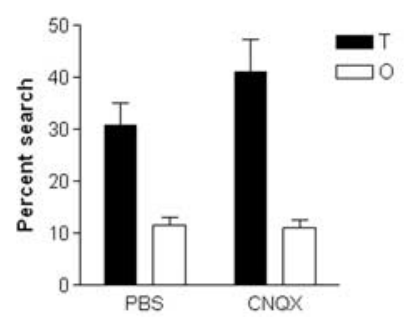

C.

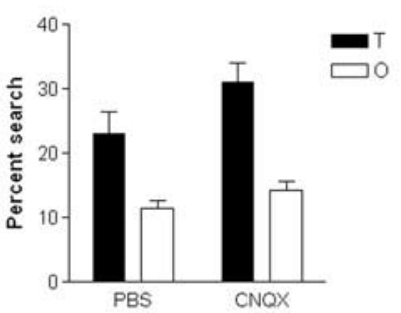

d.
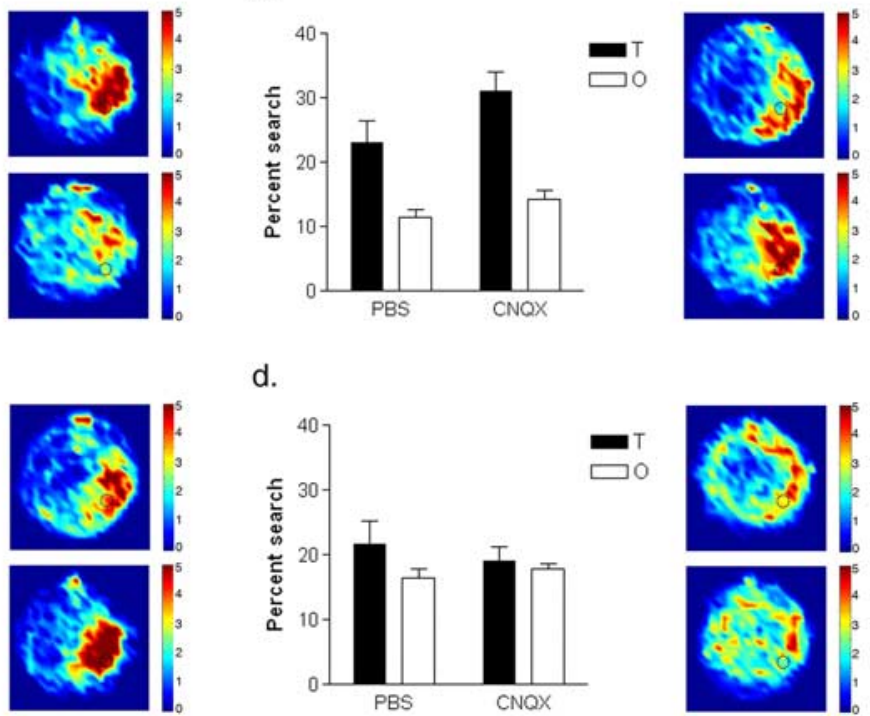

e.
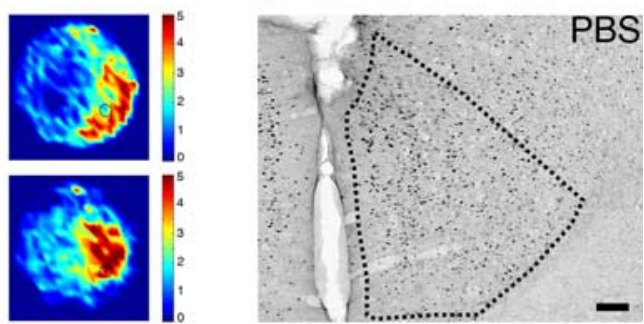

f.

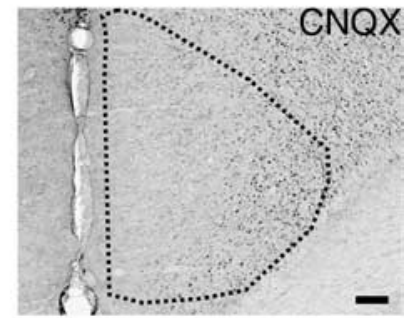

Figure 6. Effects of CNQX inactivation of ACC on remote spatial memory. In this experiment, mice were trained in the hidden version of the water maze, and their spatial preference evaluated in a series of probe tests. Thirty days after training, mice were given a remote probe test after intra-ACC infusions of either PBS or CNQX (a). These mice were subsequently retested $4 \mathrm{~d}$ later, drug-free (b). Finally, these same mice were given two more probe tests: one in which the distal visual cues present (c), the other with these cues obscured by a black curtain (d). For each of these probe tests, the percentage of time spent searching the target zone $(\mathrm{T})$ compared with other $(0)$ zones is shown on the left. Additionally, density plots for grouped data showing where searches were concentrated in the PBS (upper) and CNQX (lower) groups are shown on the right. The color scale represents the number of visits per animal per $5 \times 5 \mathrm{~cm}$ area. Representative photomicrographs of Fos expression in the ACC after water-maze testing shown for mice pretreated with either PBS (e) or CNQX (f). Scale bars, $100 \mu \mathrm{m}$. Error bars indicate SE. 
were tested drug free. As expected, with the distal cues present, both groups of mice concentrated their search in the target zone compared with other zones (significant main effect of zone: $F_{(1,13)}=22.80 ; p<0.001$ ) (Fig. 6c). However, when these cues were obscured, neither group searched selectively (zone: $F_{(1,13)}=$ $1.46 ; p=0.25$ ) (Fig. $6 d$ ). The order of the two tests (cues present vs cues obscured) was counterbalanced, and so the lack of spatial bias in the probe test with the cues removed cannot be attributed to extinction. Rather, these results indicate that spatial performance in these probe tests depends on an allocentric representation of the extramaze distal cues.

\section{Discussion}

Our results establish a role for the ACC in the expression of remote spatial memory. Using a water-maze task, we found that increased activation of the ACC correlated with expression of remote (but not recent) spatial memory. Conversely, we found that inactivating the ACC disrupted performance only in tests of remote spatial memory. This disruption of remote memory was observed in two different mouse strains after training with either a hidden or visible platform in a constant location, and using either lidocaine or the AMPA receptor antagonist CNQX. Together, these results establish that activity in neurons intrinsic to the ACC is critical for processing remote spatial memories.

\section{The hippocampus and remote spatial memory}

Previous studies have examined the impact of post-training hippocampal lesions on water-maze memory (Bolhuis et al., 1994; Mumby et al., 1999; Sutherland et al., 2001; Clark et al., 2005a,b; Martin et al., 2005). In each of these studies, hippocampal lesions disrupted spatial memories, regardless of the interval between training and surgery. This was the case even after extensive pretraining in the water maze (Clark et al., 2005b), partial lesions of hippocampus (Clark et al., 2005a; Martin et al., 2005), when extended delays between training and surgery were used (Clark et al., 2005a), when water mazes with reduced spatial complexity were used (Clark et al., 2005a), or when reminders to aid retrieval were used (Martin et al., 2005). Similarly, we found no evidence for a graded effect on recent versus remote spatial memory after inactivation of the dorsal hippocampus, consistent with a recent study using similar inactivation approaches in rats (Broadbent et al., 2006).

This temporally ungraded pattern is distinct from the timedependent role of the hippocampus in some other tasks that also involve processing spatial information [e.g., contextual fear conditioning (Kim and Fanselow, 1992; Anagnostaras et al., 1999); 5-arm discrimination (Maviel et al., 2004)]. In particular, using similar approaches, we previously showed that the hippocampus is activated after recent, but not remote, contextual fear memory testing (Frankland et al., 2004), suggesting that the hippocampus plays only a temporary role in the expression of contextual fear memories. In contrast, our gene expression and inactivation studies suggest that the hippocampus is continuously needed for the expression of a water-maze memory. There are perhaps two key differences between the water maze and contextual fear conditioning that may account for this discrepancy.

First, the hippocampus may be important for the expression of spatial memories that require animals to navigate through space, as in the water maze. In such tasks, hippocampal activity may be required to continuously integrate both idiothetic (selfmotion cues) and allothetic (e.g., distal visual cues) information to successfully navigate to the escape platform (for discussion, see Clark et al., 2005a; Martin et al., 2005). Consistent with this, we found robust hippocampal Fos expression in both the visible/ fixed and visible/variable groups, suggesting that navigational demands of the water maze alone significantly engage the hippocampus. Presumably, abolition of this activity interferes with the expression of remote (as well as recent) water maze memories, in part, by affecting the animal's ability to integrate idiothetic and allothetic information. Second, the hippocampus may always be necessary for rich, detailed spatial representation of environments (Rosenbaum et al., 2000; Winocur et al., 2005; Moscovitch et al., 2006), and such precise representations may be essential for the continued expression of a water-maze memory. This contrasts with contextual fear conditioning where mice tend to show generalized freezing at remote time-points (Balogh et al., 2002), suggesting that as these memories age they become less precise. The important distinction here is that although precision (and presumably hippocampal involvement) may not be necessary for the continued expression of a contextual fear memory, it is perhaps essential for the expression of a water-maze memory in which mice must remember a specific location in an otherwise relatively featureless environment. The application of pharmacological and/or genetic approaches that can temporally limit disruption to either the consolidation period or to testing will help to discriminate between these performance (navigation) and mnemonic (precision) accounts of water-maze deficits after hippocampal inactivation (Riedel et al., 1999; Wang et al., 2003; Cui et al., 2004; Micheau et al., 2004).

\section{The ACC and remote spatial memory}

Although we found that the hippocampus is always required for the expression of water-maze spatial memories, our key finding is that as these memories mature they become additionally dependent on the ACC. The effects of ACC inactivation on memory expression were selective, not only in terms of the remoteness of the memory to be retrieved, but also in terms of the type of memory affected. Successful performance in a probe test depends on a number of potentially dissociable memory systems (Micheau et al., 2004); an animal must remember both where to search and also how to search. More detailed analysis of probe test performance showed that ACC inactivation appeared to spare more procedural (how to) aspects of water maze memory. For example, lidocaine-treated mice did not behave like naive mice in the remote probe test. Rather, these mice still spent substantially less time in the periphery of the pool than they did at the beginning of training, indicating that memory for certain strategies (such as avoiding the walls of the pool) was not affected. In addition, lidocaine inactivation of the ACC did not impair the ability of mice to swim to a marked platform, indicating that these infusions did not interfere with sensory and motivational requirements of this task.

These data begin to identify a set of regions that play essential roles in the continued expression of a spatial memory, and suggest that circuits supporting expression of these spatial memories are reorganized in a time-dependent manner. We found that eliminating activity in the AHPC or the ACC completely disrupted expression of remote spatial memory. Also likely to play an essential role is the entorhinal cortex (Remondes and Schuman, 2004; Steffenach et al., 2005), a region that sits at the interface between the hippocampus and neocortex. For example, lesions of the dorsolateral band of the entorhinal cortex disrupt expression of a previously acquired spatial memory (Steffenach et al., 2005). These regions participate in what is very likely to be a much broader forebrain network that contributes to different aspects of spatial cognition. For example, this network may in- 
clude the retrosplenial and parietal cortices, regions that play important roles in representing spatial information in allocentric and egocentric frameworks, respectively (Moscovitch et al., 2005). In addition, this network may include the infralimbic and prelimbic cortices because neural activity in these regions correlates with motivational aspects of performance (e.g., representation of goal locations) (Hok et al., 2005). However, whereas inactivation of the ACC or dHPC completely abolished remote spatial memories, lesions of the retrosplenial, parietal, infralimbic, and prelimbic regions typically only have mild effects on spatial behavior (Warburton et al., 1998; Aggleton et al., 2000; Hoh et al., 2003). This suggests that the ACC and the hippocampus may play more essential roles in distributed forebrain networks supporting remote spatial memory.

The role identified here for the ACC in remote spatial memory parallels its role in remote memory for other types of information (Frankland and Bontempi, 2005). For example, the ACC is activated after remote memory recall in a number of tasks (Bontempi et al., 1999; Frankland et al., 2004; Maviel et al., 2004). Conversely, inactivation of the ACC disrupts recall of remote fivearm discrimination (Maviel et al., 2004) and contextual fear (Frankland et al., 2004) memories, and lesions of the mPFC (that include the ACC and prelimbic cortex) block remote (month old) trace eye-blink memories (Takehara et al., 2003). The present results extend this list to include a purely spatial memory; one that requires the mouse to navigate to a fixed point in space using an allocentric representation of a remotely learned environment.

The exact nature of the role the ACC plays in remote memory is less clear, however. One outstanding issue is whether the ACC is important for memory storage or memory retrieval. The ACC is a functionally heterogeneous structure, and activity in the ACC is related to a number of cognitive [e.g., error detection, effortful recall, attention (Carter et al., 1999; Miller, 2000)], motor [e.g., preparation and execution of movement (Paus, 2001)], and sensory [e.g., pain (Price, 2000; Zhuo, 2005)] functions. Although a small proportion of cells within the dorsal ACC exhibit place fields (Hok et al., 2005), the role of the ACC in remote memory appears to be conserved across tasks, regardless of whether they are spatial or nonspatial. The ACC is highly interconnected to other prefrontal regions, and is reciprocally connected to sensory, motor, and limbic cortices (Uylings et al., 2003; Jones et al., 2005). This connectivity places the ACC in a hub-like position, and therefore raises the possibility that this region coordinates retrieval of remote memories stored in distributed cortical networks.

\section{References}

Aggleton JP, Vann SD, Oswald CJ, Good M (2000) Identifying cortical inputs to the rat hippocampus that subserve allocentric spatial processes: a simple problem with a complex answer. Hippocampus 10:466-474.

Anagnostaras SG, Maren S, Fanselow MS (1999) Temporally graded retrograde amnesia of contextual fear after hippocampal damage in rats: within-subjects examination. J Neurosci 19:1106-1114.

Balogh SA, Radcliffe RA, Logue SF, Wehner JM (2002) Contextual and cued fear conditioning in C57BL/6J and DBA/2J mice: context discrimination and the effects of retention interval. Behav Neurosci 116:947-957.

Bolhuis JJ, Stewart CA, Forrest EM (1994) Retrograde amnesia and memory reactivation in rats with ibotenate lesions to the hippocampus or subiculum. Q J Exp Psychol B 47:129-150.

Bontempi B, Laurent-Demir C, Destrade C, Jaffard R (1999) Timedependent reorganization of brain circuitry underlying long-term memory storage. Nature 400:671-675.

Broadbent NJ, Squire LR, Clark RE (2006) Reversible hippocampal lesions disrupt water maze performance during both recent and remote memory tests. Learn Mem 13:187-191.

Burgess N, Maguire EA, O’Keefe J (2002) The human hippocampus and spatial and episodic memory. Neuron 35:625-641.

Carter CS, Botvinick MM, Cohen JD (1999) The contribution of the anterior cingulate cortex to executive processes in cognition. Rev Neurosci 10:49-57.

Cave CB, Squire LR (1991) Equivalent impairment of spatial and nonspatial memory following damage to the human hippocampus. Hippocampus 1:329-340.

Clark RE, Broadbent NJ, Squire LR (2005a) Hippocampus and remote spatial memory in rats. Hippocampus 15:260-272.

Clark RE, Broadbent NJ, Squire LR (2005b) Impaired remote spatial memory after hippocampal lesions despite extensive training beginning early in life. Hippocampus 15:340-346.

Crawley JN, Belknap JK, Collins A, Crabbe JC, Frankel W, Henderson N, Hitzemann RJ, Maxson SC, Miner LL, Silva AJ, Wehner JM, WynshawBoris A, Paylor R (1997) Behavioral phenotypes of inbred mouse strains: implications and recommendations for molecular studies. Psychopharmacology (Berl) 132:107-124.

Cui Z, Wang H, Tan Y, Zaia KA, Zhang S, Tsien JZ (2004) Inducible and reversible NR1 knockout reveals crucial role of the NMDA receptor in preserving remote memories in the brain. Neuron 41:781-793.

Day M, Langston R, Morris RG (2003) Glutamate-receptor-mediated encoding and retrieval of paired-associate learning. Nature 424:205-209.

de Hoz L, Martin SJ, Morris RG (2004) Forgetting, reminding, and remembering: the retrieval of lost spatial memory. PLoS Biol 2:E225.

Eichenbaum H, Stewart C, Morris RG (1990) Hippocampal representation in place learning. J Neurosci 10:3531-3542.

Frankland PW, Bontempi B (2005) The organization of recent and remote memories. Nat Rev Neurosci 6:119-130.

Frankland PW, Bontempi B, Talton LE, Kaczmarek L, Silva AJ (2004) The involvement of the anterior cingulate cortex in remote contextual fear memory. Science 304:881-883.

Gusev PA, Cui C, Alkon DL, Gubin AN (2005) Topography of Arc/Arg3.1 mRNA expression in the dorsal and ventral hippocampus induced by recent and remote spatial memory recall: dissociation of CA3 and CA1 activation. J Neurosci 25:9384-9397.

Hoh TE, Kolb B, Eppel A, Vanderwolf CH, Cain DP (2003) Role of the neocortex in the water maze task in the rat: a detailed behavioral and Golgi-Cox analysis. Behav Brain Res 138:81-94.

Hok V, Save E, Lenck-Santini PP, Poucet B (2005) Coding for spatial goals in the prelimbic/infralimbic area of the rat frontal cortex. Proc Natl Acad Sci USA 102:4602-4607.

Jones BF, Groenewegen HJ, Witter MP (2005) Intrinsic connections of the cingulate cortex in the rat suggest the existence of multiple functionally segregated networks. Neuroscience 133:193-207.

Kaczmarek L, Robertson HA (2002) Handbook of chemical neuroanatomy. Amsterdam: Elsevier.

Kim JJ, Fanselow MS (1992) Modality-specific retrograde amnesia of fear. Science 256:675-677.

Leutgeb S, Leutgeb JK, Moser MB, Moser EI (2005) Place cells, spatial maps and the population code for memory. Curr Opin Neurobiol 15:738-746.

Maguire EA, Burke T, Phillips J, Staunton H (1996) Topographical disorientation following unilateral temporal lobe lesions in humans. Neuropsychologia 34:993-1001.

Martin SJ, de Hoz L, Morris RG (2005) Retrograde amnesia: neither partial nor complete hippocampal lesions in rats result in preferential sparing of remote spatial memory, even after reminding. Neuropsychologia 43:609-624.

Maviel T, Durkin TP, Menzaghi F, Bontempi B (2004) Sites of neocortical reorganization critical for remote spatial memory. Science 305:96-99.

Micheau J, Riedel G, Roloff EL, Inglis J, Morris RG (2004) Reversible hippocampal inactivation partially dissociates how and where to search in the water maze. Behav Neurosci 118:1022-1032.

Miller EK (2000) The prefrontal cortex and cognitive control. Nat Rev Neurosci 1:59-65.

Morris RG, Garrud P, Rawlins JN, O’Keefe J (1982) Place navigation impaired in rats with hippocampal lesions. Nature 297:681-683.

Morris RG, Moser EI, Riedel G, Martin SJ, Sandin J, Day M, O'Carroll C (2003) Elements of a neurobiological theory of the hippocampus: the 
role of activity-dependent synaptic plasticity in memory. Philos Trans R Soc Lond B Biol Sci 358:773-786.

Moscovitch M, Rosenbaum RS, Gilboa A, Addis DR, Westmacott R, Grady C, McAndrews MP, Levine B, Black S, Winocur G, Nadel L (2005) Functional neuroanatomy of remote episodic, semantic and spatial memory: a unified account based on multiple trace theory. J Anat 207:35-66.

Moscovitch M, Nadel L, Winocur G, Gilboa A, Rosenbaum RS (2006) The cognitive neuroscience of remote episodic, semantic and spatial memory. Curr Opin Neurobiol 16:179-190.

Moser E, Moser MB, Andersen P (1993) Spatial learning impairment parallels the magnitude of dorsal hippocampal lesions, but is hardly present following ventral lesions. J Neurosci 13:3916-3925.

Moser MB, Moser EI (1998) Distributed encoding and retrieval of spatial memory in the hippocampus. J Neurosci 18:7535-7542.

Mumby DG, Astur RS, Weisend MP, Sutherland RJ (1999) Retrograde amnesia and selective damage to the hippocampal formation: memory for places and object discriminations. Behav Brain Res 106:97-107.

Nakazawa K, McHugh TJ, Wilson MA, Tonegawa S (2004) NMDA receptors, place cells and hippocampal spatial memory. Nat Rev Neurosci 5:361-372.

O'Keefe JM, Nadel L (1978) The hippocampus as a cognitive map. Oxford: Oxford UP.

Paus T (2001) Primate anterior cingulate cortex: where motor control, drive and cognition interface. Nat Rev Neurosci 2:417-424.

Paxinos G, Franklin KBJ (2000) The mouse brain in stereotaxic coordinates, Ed 2. San Diego: Academic.

Price DD (2000) Psychological and neural mechanisms of the affective dimension of pain. Science 288:1769-1772.

Remondes M, Schuman EM (2004) Role for a cortical input to hippocampal area CA1 in the consolidation of a long-term memory. Nature 431:699-703.

Riedel G, Micheau J, Lam AG, Roloff E, Martin SJ, Bridge H, Hoz L, Poeschel B, McCulloch J, Morris RG (1999) Reversible neural inactivation reveals hippocampal participation in several memory processes. Nat Neurosci 2:898-905.

Rosenbaum RS, Priselac S, Kohler S, Black SE, Gao F, Nadel L, Moscovitch M (2000) Remote spatial memory in an amnesic person with extensive bilateral hippocampal lesions. Nat Neurosci 3:1044-1048.

Rosenbaum RS, Ziegler M, Winocur G, Grady CL, Moscovitch M (2004) "I have often walked down this street before": fMRI studies on the hippocampus and other structures during mental navigation of an old environment. Hippocampus 14:826.
Sandkuhler J, Maisch B, Zimmermann M (1987) The use of local anaesthetic microinjections to identify central pathways: a quantitative evaluation of the time course and extent of the neuronal block. Exp Brain Res 68:168-178.

Smith ML, Milner B (1981) The role of the right hippocampus in the recall of spatial location. Neuropsychologia 19:781-793.

Squire LR, Alvarez P (1995) Retrograde amnesia and memory consolidation: a neurobiological perspective. Curr Opin Neurobiol 5:169-177.

Squire LR, Clark RE, Knowlton BJ (2001) Retrograde amnesia. Hippocampus 11:50-55.

Steffenach HA, Witter M, Moser MB, Moser EI (2005) Spatial memory in the rat requires the dorsolateral band of the entorhinal cortex. Neuron 45:301-313.

Sutherland RJ, Weisend MP, Mumby D, Astur RS, Hanlon FM, Koerner A, Thomas MJ, Wu Y, Moses SN, Cole C, Hamilton DA, Hoesing JM (2001) Retrograde amnesia after hippocampal damage: recent vs. remote memories in two tasks. Hippocampus 11:27-42.

Takehara K, Kawahara S, Kirino Y (2003) Time-dependent reorganization of the brain components underlying memory retention in trace eyeblink conditioning. J Neurosci 23:9897-9905.

Teng E, Squire LR (1999) Memory for places learned long ago is intact after hippocampal damage. Nature 400:675-677.

Uylings HB, Groenewegen HJ, Kolb B (2003) Do rats have a prefrontal cortex? Behav Brain Res 146:3-17.

Wang H, Shimizu E, Tang YP, Cho M, Kyin M, Zuo W, Robinson DA, Alaimo PJ, Zhang C, Morimoto H, Zhuo M, Feng R, Shokat KM, Tsien JZ (2003) Inducible protein knockout reveals temporal requirement of CaMKII reactivation for memory consolidation in the brain. Proc Natl Acad Sci USA 100:4287-4292.

Warburton EC, Aggleton JP, Muir JL (1998) Comparing the effects of selective cingulate cortex lesions and cingulum bundle lesions on water maze performance by rats. Eur J Neurosci 10:622-634.

Winocur G, Moscovitch M, Fogel S, Rosenbaum RS, Sekeres M (2005) Preserved spatial memory after hippocampal lesions: effects of extensive experience in a complex environment. Nat Neurosci 8:273-275.

Wolfer DP, Stagljar-Bozicevic M, Errington ML, Lipp HP (1998) Spatial memory and learning in transgenic mice: fact or artifact? News Physiol Sci 13:118-123.

Zhuo M (2005) Targeting central plasticity: a new direction of finding painkillers. Curr Pharm Des 11:2797-2807. 